\title{
Application of Economic MPC to Frequency Control in a Single-Area
}

\section{Sokoler, Leo Emil; Edlund, Kristian; Jørgensen, John Bagterp}

Published in:

Proceedings of the 54th IEEE Conference on Decision and Control (CDC 2015)

Link to article, DOI:

10.1109/CDC.2015.7402613

Publication date:

2016

Document Version

Peer reviewed version

Link back to DTU Orbit

Citation (APA):

Sokoler, L. E., Edlund, K., \& Jørgensen, J. B. (2016). Application of Economic MPC to Frequency Control in a Single-Area. In Proceedings of the 54th IEEE Conference on Decision and Control (CDC 2015) (pp. 2635-2642). IEEE. https://doi.org/10.1109/CDC.2015.7402613

\section{General rights}

Copyright and moral rights for the publications made accessible in the public portal are retained by the authors and/or other copyright owners and it is a condition of accessing publications that users recognise and abide by the legal requirements associated with these rights.

- Users may download and print one copy of any publication from the public portal for the purpose of private study or research.

- You may not further distribute the material or use it for any profit-making activity or commercial gain

- You may freely distribute the URL identifying the publication in the public portal 


\title{
Application of Economic MPC to Frequency Control in a Single-Area Power System
}

\author{
Leo Emil Sokoler, Kristian Edlund and John Bagterp Jørgensen
}

\begin{abstract}
This paper presents a novel model predictive control scheme for frequency control in a single-area power system. The proposed controller provides set-point corrections to the system power generators, based on the solution to an optimal control problem. The optimal control problem directly incorporates the cost of operation into its objective function. A trade-off parameter is used to balance set-point tracking and cost minimization. Simulations based on a Faroe Islands case study show that the proposed approach reduces cost of operation by almost an order of magnitude, compared to both set-point based model predictive control as well as conventional frequency-based PI-control.
\end{abstract}

\section{INTRODUCTION}

Power production planning is an important task in power system operations. The task involves solving a mixed-integer optimization problem for unit commitment and economic dispatch of the system power generators [1], [2]. This optimization problem is a computationally challenging problem that may take up to several minutes, or even hours, to solve. To compensate for real-time fluctuations in the power production and the power consumption, a second control layer is used. This layer is responsible for the activation of operational reserves. Planning the operational reserves is an integral part of the unit commitment problem. In this paper, we refer to the solution of the unit commitment problem as the nominal production plan.

In small isolated power systems, a single operator is often responsible for both power transmission and power production. An example of such an isolated power system is the Faroe Islands. Here the municipality-owned company SEV acts both as the transmission system operator (TSO) and as the sole power generating company. This means that SEV is responsible for balancing production and consumption, including the activation of operational reserves.

In the Faroe Islands, the operational reserves can be categorized into two main categories: automatic reserves and manual reserves. The automatic reserves are frequency controlled reserves that are activated in direct proportion to frequency deviations from the nominal frequency (primary control). Primary control stabilizes the frequency at a steadystate that deviates from the nominal frequency. The manual reserves are activated to eliminate the steady-state error, such that the frequency is returned to its nominal value (secondary control). Secondary control is also known as load frequency control (LFC). Following the activation of manual reserves,

L. E. Sokoler and J. B. Jørgensen are affiliated with the Department of Applied Mathematics and Computer Science, Technical University of Denmark, DK-2800 Kgs. Lyngby, Denmark $\{$ leso, jb jo $\}$ @ dtu.dk

K. Edlund are affiliated with DONG Energy, DK-2830 Virum, Denmark. a re-dispatch of the generating units may be performed to free up the required operational reserves.

Activating reserves is associated with a cost. Some generators have a low marginal production cost (e.g. wind turbines and hydro turbines), and others have a high marginal production cost (e.g. diesel generators and gas turbines). While this information is accounted for in the unit commitment problem, it is often neglected by the controllers that activate the operational reserves. An approximate method to use information on the reserve activation cost, is to combine a PI-control structure for LFC with so-called participation factors [3]-[6]. The participation factor of a generator is a gain that determines its degree of participation in the LFC. The participation factors do not distinguish between up and down regulation, which is a significant drawback from an economical point of view. Moreover, the approach does not consider the frequency dynamics. As an example, it is desirable to activate fast but expensive power generators in situations where the frequency drops significantly below the nominal frequency. Conversely, it is attractive to activate cheaper generators when the frequency drop is less significant.

In this paper, we use economic model predictive control (EMPC) [7], [8] in a novel way to active operational reserves in an isolated power system. The reserves are activated based on the solution to an optimal control problem (OCP), which takes into account real-time measurements, and updated forecasts of e.g. renewable energy production. Reference [9] provides an example of short-term forecasts that can be used for improved frequency control. The OCP objective function is formulated as a bi-objective criterion that trades off the cost of operation and set-point tracking.

Set-point based MPC have been considered for LFC in [10]-[12], and for tertiary control in [13]. References [14][16] develop distributed algorithms for such conventional MPC schemes. In the previous work [10]-[16], quadratic penalty functions are used to ensure 1) that the load flows on the tie-lines to other areas are restored to their scheduled values, and 2) that the frequency is returned to its nominal value. References [10] and [12] include an inputrate regularization term in the OCP objective function, to reduce wear and tear on the power generators. The main novelty of this paper, is to introduce a generalized OCP that directly incorporates the cost of operation into the MPC layer. Moreover, while existing work focus on multi-area and interconnected power systems, the proposed EMPC scheme is tailored to isolated power systems. In such systems, no power is exchanged with neighboring regions and no 


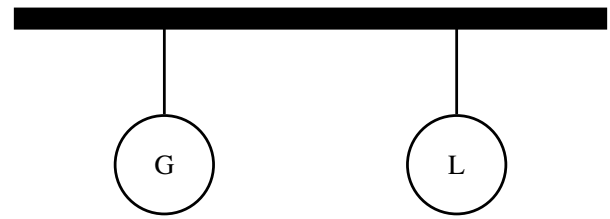

Fig. 1. Schematic diagram of the single-area power system. The system consists of a bus, which is connected to a number of generators (G), and an aggregation of loads (L).

markets are available for trading energy. The emphasis on isolated power systems is motivated by the GRANI project [17]. The GRANI project is a collaboration between DONG Energy and the Faroe Islands. The Faroe Islands acts as a live demonstration laboratory for testing new power system control technologies.

To test the proposed EMPC scheme, a non-linear simulation model of an isolated power system is developed. We linearize the non-linear model for prediction and control. To estimate non-predictable disturbances, the linear model is augmented by a disturbance model. Simulations are performed using a case study based on the Faroe Islands' power system. We compare EMPC to set-point based MPC and frequency-based PI-control. Set-point based MPC occurs as a special case of the proposed EMPC scheme. Simulations show that significant cost reductions can be achieved by trading off cost of operations and set-point tracking, even without compromising the high quality frequency control associated with set-point based MPC.

We have organized this paper as follows. Section II derives a non-linear simulation model of an isolated power system. Section III introduces an EMPC scheme for frequency control in this system, and Section IV presents a Faroe Islands case study. Section V concludes the paper.

\section{MODEL}

This section presents a stochastic non-linear simulation model of a small isolated power system. The system is represented by a single-area power system, in which the frequency is equal for all the power generators. The Faroe Islands is a fairly meshed system, where line capacity constraints and transmission losses are negligible for the application considered in this paper. A linearized model of the single-area power system is derived for control purpose. We provide the Kalman filter equations for state estimation in the stochastic linear system.

\section{A. Simulation Model}

Fig. 1 is a diagram of the single-area power system. The system consists of three main components: a collection of power generators, a load, and a bus. By convention, negative sign is used for power consumption and positive sign is used for power production.

Power generators are modeled with different levels of detail, depending on the application of interest. Linear models are often well suited to describe the relation between generator power set-point, and generator power production
[4], [5], [18]-[21]. Reference [22] validates such linear models against actual measurement data. Similar models have been used for MPC in [10]-[12], [15], [16], [18].

In this paper, a collection of $M$ power generators is modeled by the linear state space model

$$
\begin{aligned}
\dot{x}_{g_{i}}(t) & =A_{g_{i}} x_{g_{i}}(t)+B_{g_{i}} u_{g_{i}}(t), & & i \in \mathcal{M}, \\
z_{g_{i}}(t) & =C_{g_{i}} x_{g_{i}}(t), & & i \in \mathcal{M},
\end{aligned}
$$

with $\mathcal{M}=\{1,2, \ldots, M\}$. In Equation (1), $u_{g_{i}}(t)$ is the power set-point of generator $i, x_{g_{i}}(t)$ is the state of generator $i$, and $z_{g_{i}}(t)$ is the power production of generator $i$.

The power set-point, $u_{g_{i}}(t)$, is separated into the following two components

$$
u_{g_{i}}(t)=\underbrace{\tilde{u}_{g_{i}}(t)}_{\text {System Level }}-\underbrace{K_{i}\left(z_{f}(t)-f_{0}\right)}_{\text {Local Level }}, \quad i \in \mathcal{M} .
$$

The system level control component is determined at a centralized level, in which interactions between the power generators are accounted for. This component includes the nominal set-point, as well as set-point adjustments resulting from secondary control. The local level control component models the primary control of each power generator. Primary control is activated in direct proportion to frequency deviations from the nominal frequency [5], [20], [21]. The nominal frequency is denoted $f_{0}$, the current frequency is denoted $z_{f}(t)$, and the proportional gain associated with the primary control of generator $i$ is denoted $K_{i}$. The model (1) is valid for set-points in the the interval $\underline{u}_{g_{i}}(t) \leq u_{g_{i}}(t) \leq$ $\bar{u}_{g_{i}}(t)$. The parameter $\underline{u}_{g_{i}}(t)$ is the minimum production of generator $i$, and $\bar{u}_{g_{i}}(t)$ is the maximum production of generator $i$.

The load in Fig. 1 represents an aggregate of all the loads in the system. The aggregate may include the power production of non-controllable power generators, such as non-controllable wind-turbines and solar cells. We model the load using a linear state space model in the form

$$
\begin{aligned}
\dot{x}_{l}(t) & =A_{l} x_{l}(t)+B_{l} d_{l}(t), \\
z_{l}(t) & =C_{l} x_{l}(t),
\end{aligned}
$$

The input $d_{l}(t)$ is the load set-point, $x_{l}(t)$ is the load state, and $z_{l}(t)$ is the actual load. Later in this paper, the load set-point is replaced by a piecewise constant load forecast. Modeling the load using the filtered value $z_{l}(t)$, instead of the load forecast, better represents the physical behavior of the system, since the load does not change instantaneously.

The power balance at the bus is

$$
\begin{aligned}
z_{b}(t) & =\sum_{i \in \mathcal{M}} z_{g_{i}}(t)+z_{l}(t) \\
& =\sum_{i \in \mathcal{M}} C_{g_{i}} x_{g_{i}}(t)+C_{l} x_{l}(t),
\end{aligned}
$$

Using the swing equation for a synchronous machine [5], [20], [21], the following model for the system frequency is derived

$$
\begin{aligned}
\dot{x}_{f}(t) & =A_{f}\left(x_{f}(t)\right) z_{b}(t), \\
z_{f}(t) & =x_{f}(t)
\end{aligned}
$$


where $z_{f}(t)$ is the system frequency, and

$$
A_{f}\left(x_{f}(t)\right)=f_{0}^{2} /\left(2 H S x_{f}(t)\right) .
$$

Note that (5) is a non-linear system, since $A_{f}\left(x_{f}(t)\right)$ is a function of the system frequency. In Equation (6)

$$
H=\sum_{i \in \mathcal{M}} H_{i} S_{i} / S, \quad S=\sum_{i \in \mathcal{M}} S_{i} .
$$

Generator $i$ has constant of inertia $H_{i}$ and rating $S_{i}$. Reference [23] lists these values for different types of generators.

Collect the generator subsystems (1) into a single linear state space model with block-angular matrices $\left(A_{g}, B_{g}, C_{g}\right)$, such that $x_{g}=\left[x_{g_{1}}, x_{g_{2}}, \ldots, x_{g_{M}}\right]$, and similarly introduce $u_{g}$ and $z_{g}$. Also define the frequency deviation variables

$$
z_{\Delta f}(t)=x_{\Delta f}(t)=z_{f}(t)-f_{0}
$$

Equations (1), (2), (3), (4) and (5) are combined to form the system model

$$
\begin{aligned}
\dot{x}(t) & =f(x(t), u(t), d(t)), \\
z(t) & =g(x(t)),
\end{aligned}
$$

$u(t)=\tilde{u}_{g}(t), x(t)=\left[x_{g}(t)^{T}, x_{l}(t)^{T}, x_{\Delta f}(t)\right]^{T}, z(t)=$ $\left[z_{g}(t)^{T}, z_{l}(t), z_{b}(t), z_{\Delta f}(t)\right]^{T}$, and $d(t)=d_{l}(t)$. Define the vector function

$$
L(x(t))=\left[\begin{array}{ccc}
A_{g} & 0 & -B_{g} K \\
0 & A_{l} & 0 \\
A_{\Delta f}(x(t)) e^{T} C_{g} & A_{\Delta f}(x(t)) C_{l} & 0
\end{array}\right] x(t),
$$

where $e$ is a vector of all ones, $K=\left[K_{1}, K_{2}, \ldots, K_{M}\right]^{T}$, and $A_{\Delta f}(x(t))=A_{f}\left(f_{0}+x_{\Delta f}(t)\right)$. Moreover, define the matrices

$$
B=\left[\begin{array}{c}
B_{g} \\
0 \\
0
\end{array}\right], E=\left[\begin{array}{c}
0 \\
B_{l} \\
0
\end{array}\right], C_{z}=\left[\begin{array}{ccc}
C_{g} & 0 & 0 \\
0 & C_{l} & 0 \\
e^{T} C_{g} & C_{l} & 0 \\
0 & 0 & 1
\end{array}\right] .
$$

Using these definitions, the system model may be written in the form (7) with

$$
\begin{aligned}
f(x(t), u(t), d(t)) & =L(x(t))+B u(t)+E d(t), \\
g(x(t)) & =C_{z} x(t) .
\end{aligned}
$$

The deterministic model is augmented by stochastic terms. The stochastic model is

$$
\begin{aligned}
\boldsymbol{x}\left(t_{k}+T_{s}\right) & =F\left(\boldsymbol{x}\left(t_{k}\right), u\left(t_{k}\right)+\boldsymbol{w}_{u}\left(t_{k}\right),\right. \\
& \left.d\left(t_{k}\right)+b\left(t_{k}\right)+\boldsymbol{w}_{d}\left(t_{k}\right)\right), \\
\boldsymbol{y}\left(t_{k}\right) & =h\left(\boldsymbol{x}\left(t_{k}\right)\right)+\boldsymbol{v}\left(t_{k}\right), \\
\boldsymbol{z}\left(t_{k}\right) & =g\left(\boldsymbol{x}\left(t_{k}\right)\right) .
\end{aligned}
$$

$\boldsymbol{y}\left(t_{k}\right)$ is a vector of measurements, $\boldsymbol{w}_{u}\left(t_{k}\right)$ is the generator process noise, $\boldsymbol{w}_{d}\left(t_{k}\right)$ is the load process noise, and $\boldsymbol{v}\left(t_{k}\right)$ is the measurement noise. Subscript $k$ refers to a fixed time index. The available measurements are the power production of each generator, the power balance at the bus, and the system frequency. This means that $h(\boldsymbol{x}(t))=C_{y} \boldsymbol{x}(t)$, where $C_{y}$ is a sub-matrix of $C_{z}$. The sampling time is denoted $T_{s}$.

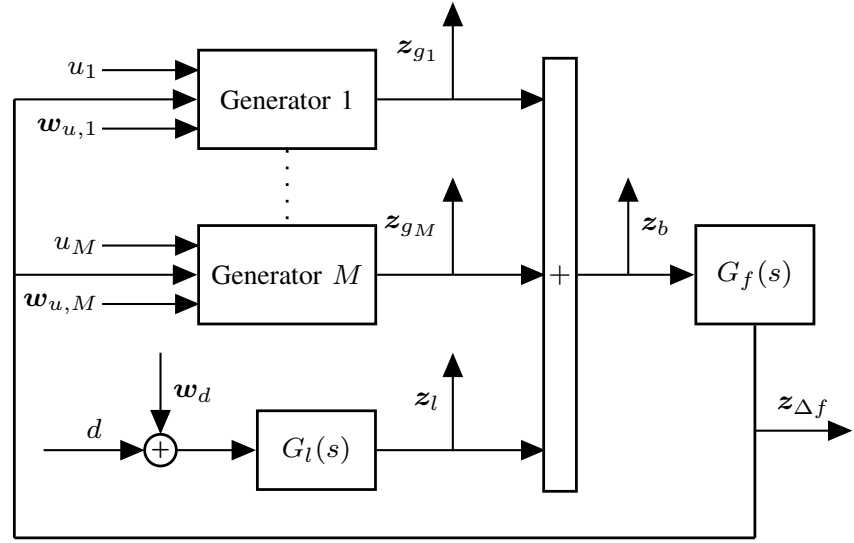

Fig. 2. System dynamics in the linear stochastic model (10). The transfer functions $G_{l}(s)$ and $G_{f}(s)$ represent the load and linearized frequency dynamics, respectively.

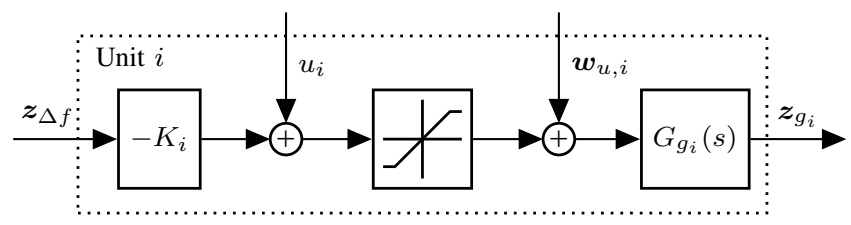

Fig. 3. Generator dynamics in the linear stochastic model (10). The transfer function $G_{g_{i}}(s)$ represents the dynamics of generator $i$.

We use $T_{s}=0.1 \mathrm{~s}$. Bold letters indicate random variables. The system noise is assumed to consist of independent and identically distributed random variables with $\boldsymbol{w}_{u}\left(t_{k}\right) \sim$ $N\left(0, R_{w_{u}}\right), \boldsymbol{w}_{d}\left(t_{k}\right) \sim N\left(0, R_{w_{d}}\right)$, and $\boldsymbol{v}\left(t_{k}\right) \sim N\left(0, R_{v}\right)$.

In the stochastic model (9), $d\left(t_{k}\right)$ is interpreted as a piecewise constant load forecast. The parameters $b\left(t_{k}\right)$ and $\boldsymbol{w}_{d}\left(t_{k}\right)$ account for the forecast errors. The term $b\left(t_{k}\right)$ models unpredictable disturbances due to e.g. generator trips and non-zero mean forecast errors, and the term $\boldsymbol{w}_{d}\left(t_{k}\right)$ accounts for random fluctuations from the mean. We simulate the system using ode 45 in MATLAB.

\section{B. Control Model}

The controller proposed in this paper keeps the system frequency, $z_{f}(t)$, close to its nominal value, $f_{0}$. Equation (6) shows that when $x_{f}(t) \approx f_{0}$, then $A_{f}\left(x_{f}(t)\right) \approx f_{0} /(2 H S)$. It follows that $L(x(t)) \approx A x(t)$, with $A$ defined accordingly. For the application presented in this paper, it is therefore adequate to use a linearization of (9) in the controller. Fig. 2 and Fig. 3 provide a block-diagram of the linearized system. The saturation block in Fig. 3 illustrates that the generator model is valid only for set-points within the generator production limits.

With some abuse of notation, the linearized system is written as

$$
\begin{aligned}
\boldsymbol{x}_{k+1} & =A \boldsymbol{x}_{k}+B\left(u_{k}+\boldsymbol{w}_{u, k}\right)+E\left(d_{k}+\boldsymbol{w}_{d, k}\right), \\
\boldsymbol{y}_{k} & =C_{y} \boldsymbol{x}_{k}+\boldsymbol{v}_{k}, \\
\boldsymbol{z}_{k} & =C_{z} \boldsymbol{x}_{k},
\end{aligned}
$$

where $(A, B, E)$ have been redefined to denote discretetime state space matrices. These matrices are computed from 
the continuous-time state space matrices using the matrix exponential. The matrices $C_{z}$ and $C_{y}$ are not redefined. The disturbance $b\left(t_{k}\right)$ is not included in (10a), as this parameter is unknown to the controller. Assuming that the sampling time is $T_{s}=0.1$ as in the continuous-time case, then $\boldsymbol{w}_{u, k} \sim N\left(0, R_{w_{u}}\right), \boldsymbol{w}_{d, k} \sim N\left(0, R_{w_{d}}\right)$, and $\boldsymbol{v}_{k} \sim$ $N\left(0, R_{v}\right)$. We derive a representation of (10), with sampling time $\bar{T}_{s}=n T_{s}=n 0.1$, for some integer $n \geq 1$. Increasing the sampling time may be necessary to accommodate the proposed controller to an existing control system, or to reduce the computation time of solving the OCP.

When $\bar{T}_{s}=n T_{s}$, the control input, $u_{k}$, is constant for $k n T_{s} \leq t \leq(k+1) n T_{s}$. The controller assumes that the demand forecast, $d_{k}$, is constant in this interval as well. Average values can be fed into the controller if this is not the case.

Using Equation (10a), the state evolution from time $t=$ $k n T_{s}$ to time $t=(k+1) n T_{s}$, is

$$
\boldsymbol{x}_{(k+1) n}=\tilde{A} \boldsymbol{x}_{k n}+\tilde{B} u_{k n}+\tilde{E} d_{k n}+\tilde{\boldsymbol{w}}_{k n},
$$

where

$$
\tilde{A}=A^{n}, \quad \tilde{B}=\sum_{i=1}^{n} A^{i-1} B, \quad \tilde{E}=\sum_{i=1}^{n} A^{i-1} E .
$$

In addition, $\tilde{\boldsymbol{w}}_{k n} \sim N\left(0, \tilde{R}_{w}\right)$, with covariance matrix

$$
\tilde{R}_{w}=\sum_{i=1}^{n} A^{k-1}\left(B R_{w_{u}} B^{T}+E R_{w_{d}} E^{T}\right)\left(A^{k-1}\right)^{T} .
$$

By letting $k:=n k$, Equations (10b), (10c), and (11) provide a discrete-time linear state space model for the linearization of (9), with sampling time $\bar{T}_{s}=n T_{s}$.

\section{State Estimation}

The system (9) is a stochastic system. We estimate the system state using the Kalman filter [24]. The Kalman filter is implemented based on the linearized model defined by $\left(\tilde{A}, \tilde{B}, \tilde{E}, C_{z}, C_{y}, \tilde{R}_{w}, R_{v}\right)$. To estimate the unknown disturbance, $b\left(t_{k}\right)$, we augment the model by a disturbance model [25], [26]. The disturbance model is

$$
\boldsymbol{\eta}_{k+1}=\boldsymbol{\eta}_{k}+\boldsymbol{w}_{\eta, k}
$$

with $\boldsymbol{w}_{\eta, k} \sim N\left(0, R_{\eta}\right)$. We denote the augmented state space system by

$$
\begin{aligned}
\boldsymbol{x}_{k+1} & =A \boldsymbol{x}_{k}+B u_{k}+E d_{k}+\boldsymbol{w}_{k}, \\
\boldsymbol{y}_{k} & =C_{y} \boldsymbol{x}_{k}+\boldsymbol{v}_{k}, \\
\boldsymbol{z}_{k} & =C_{z} \boldsymbol{x}_{k} .
\end{aligned}
$$

In this system

$$
\boldsymbol{x}_{k}:=\left[\begin{array}{l}
\boldsymbol{x}_{k} \\
\boldsymbol{\eta}_{k}
\end{array}\right], A:=\left[\begin{array}{cc}
\tilde{A} & \tilde{E} \\
0 & 1
\end{array}\right], \quad B:=\left[\begin{array}{c}
\tilde{B} \\
0
\end{array}\right], \quad E:=\left[\begin{array}{c}
\tilde{E} \\
0
\end{array}\right],
$$

$C_{z}:=\left[\begin{array}{ll}C_{z} & 0\end{array}\right]$, and $C_{y}:=\left[\begin{array}{ll}C_{y} & 0\end{array}\right]$. Finally, $\boldsymbol{w}_{k} \sim$ $N\left(0, R_{w}\right)$ with $R_{w}:=\operatorname{blkdiag}\left(\tilde{R}_{w}, R_{\eta}\right)$, using MATLAB notation.

Define $\mathcal{I}_{k}=\left\{\mathcal{I}_{k-1}, u_{k-1}, d_{k-1}, y_{k}\right\}$, with $\mathcal{I}_{0}=y_{0}$. Moreover, introduce the conditional means $\hat{x}_{k+j \mid k}=E\left[\boldsymbol{x}_{k+j} \mid \mathcal{I}_{k}\right]$,

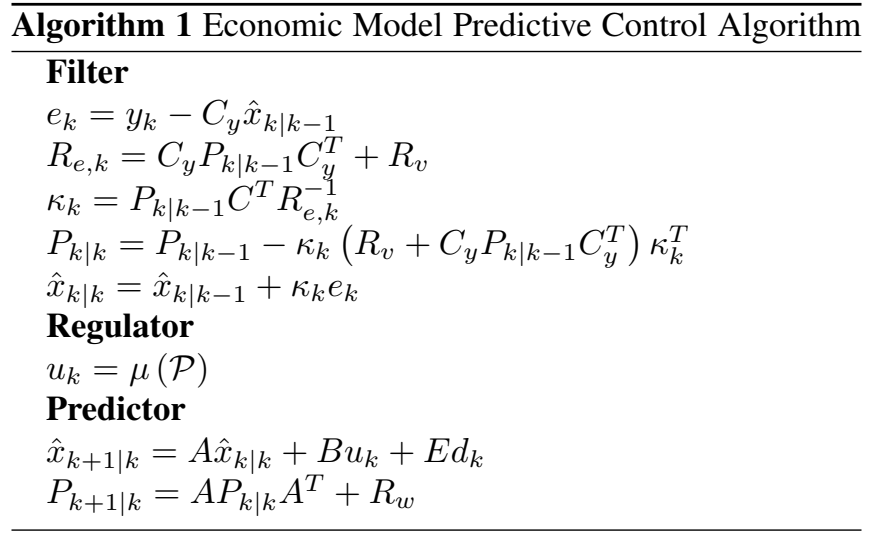

$\hat{y}_{k+j \mid k}=E\left[\boldsymbol{y}_{k+j} \mid \mathcal{I}_{k}\right], \hat{z}_{k+j \mid k}=E\left[\boldsymbol{z}_{k+j} \mid \mathcal{I}_{k}\right]$, and the conditional covariance matrix $P_{k+j \mid k}=V\left[\boldsymbol{x}_{k+j} \mid \mathcal{I}_{k}\right]$. The filtered estimate, $\hat{x}_{k \mid k}$, and the covariance matrix, $P_{k \mid k}$, is computed as

$$
\begin{aligned}
e_{k} & =y_{k}-\hat{y}_{k \mid k-1}=y_{k}-C_{y} \hat{x}_{k \mid k-1}, \\
R_{e, k} & =C_{y} P_{k \mid k-1} C_{y}^{T}+R_{v} \\
\kappa_{k} & =P_{k \mid k-1} C^{T} R_{e, k}^{-1} \\
P_{k \mid k} & =P_{k \mid k-1}-\kappa_{k} R_{e, k} \kappa_{k}^{T} \\
\hat{x}_{k \mid k} & =\hat{x}_{k \mid k-1}+\kappa_{k} e_{k},
\end{aligned}
$$

$\kappa$ is the Kalman filter gain, $e_{k}$ is the innovation, and $R_{e, k}$ is the innovation covariance matrix. The $j$-step ahead prediction for $j \geq 0$ is

$$
\begin{aligned}
\hat{x}_{k+1+j \mid k} & =A \hat{x}_{k+j \mid k}+B u_{k+j}+E d_{k+j}, \\
P_{k+1+j \mid k} & =A P_{k+j \mid k} A^{T}+R_{w} .
\end{aligned}
$$

Finally, $\hat{z}_{k+j}=C_{z} \hat{x}_{k+j \mid k}$, for $j \geq 0$.

\section{EConomic Model Predictive Control}

This section presents an EMPC scheme for controlling the single-area power system (9). Algorithm 1 list the EMPC scheme. The function $\mu$ solves the OCP, and returns the first element in the optimal input sequence $\left\{u_{k+j}^{*}\right\}_{j=0}^{N}$. The input argument $\mathcal{P}$ denotes a set of input parameters to the OCP. The OCP solved in this paper is formulated as a convex quadratic program.

\section{A. Nominal Solution}

The nominal production plan is computed by solving a unit-commitment and economic dispatch problem. To account for the nominal production plan in the EMPC scheme, we separate the inputs, states, disturbances, and outputs, in (14), into two components

$$
\begin{array}{ll}
u_{k}=u_{k}^{\mathrm{nom}}+u_{k}^{\mathrm{mpc}}, & d_{k}=d_{k}^{\mathrm{nom}}+d_{k}^{\mathrm{mpc}}, \\
x_{k}=x_{k}^{\text {nom }}+x_{k}^{\mathrm{mpc}}, & z_{k}=z_{k}^{\text {nom }}+z_{k}^{\mathrm{mpc}} .
\end{array}
$$

The input $u_{k}^{\text {nom }}$ is the pre-computed nominal set-point, and $u_{k}^{\mathrm{mpc}}$ is the set-point correction computed in real-time. Accordingly, the disturbance $d_{k}$ is partitioned into $d_{k}^{\text {nom }}$, which is known at the time the nominal set-point is computed, and $d_{k}^{\mathrm{mpc}}$, which is known only by the real-time controller. 
Using the state space model (14), with $\boldsymbol{w}_{k}=\boldsymbol{v}_{k}=0$, $u_{k}=u_{k}^{\text {nom }}$, and $d_{k}=d_{k}^{\text {nom }}$, we compute the nominal state and output values, $x_{k}^{\text {nom }}$ and $z_{k}^{\text {nom }}$, respectively. Similarly, the generator set-points that include the effect of primary control, as defined by (2), is written as

$$
u_{g, k}=u_{g, k}^{\mathrm{nom}}+u_{g, k}^{\mathrm{mpc}}
$$

where the individual components are

$$
\begin{aligned}
& u_{g, k}^{\mathrm{nom}}=\tilde{u}_{g, k}^{\mathrm{nom}}+K z_{\Delta f, k}^{\mathrm{nom}}, \\
& u_{g, k}^{\mathrm{mpc}}=\tilde{u}_{g, k}^{\mathrm{mpc}}+K z_{\Delta f, k}^{\mathrm{mpc}} .
\end{aligned}
$$

\section{B. Optimal Control Problem}

The OCP solved at every sampling time is defined as

$$
\min _{X} \phi=\sum_{j=0}^{N-1} l_{j}\left(u_{g, k+j}^{\mathrm{mpc}}, \hat{z}_{k+j+1 \mid k}^{\mathrm{mpc}}\right),
$$

subject to

$$
\begin{array}{ll}
\hat{x}_{k+j+1 \mid k}^{\mathrm{mpc}}=A \hat{x}_{k+j \mid k}^{\mathrm{mpc}}+B u_{k+j}^{\mathrm{mpc}}+E d_{k+j}^{\mathrm{mpc}}, & j \in \mathcal{N}, \\
\hat{z}_{k+j+1 \mid k}^{\mathrm{mpc}}=C_{z} \hat{x}_{k+j+1 \mid k}^{\mathrm{mpc}}, & j \in \mathcal{N}, \\
u_{g, k+j}=u_{g, k+j}^{\mathrm{nom}}+u_{g, k+j}^{\mathrm{mpc}}, & j \in \mathcal{N}, \\
u_{g, k+j}^{\mathrm{mpc}}=u_{k+j}^{\mathrm{mpc}}+K \hat{z}_{\Delta f, k+j \mid k}^{\mathrm{mpc}}, & j \in \mathcal{N}, \\
\underline{u}_{k+j} \leq u_{g, k+j} \leq \bar{u}_{k+j}, & j \in \mathcal{N} .
\end{array}
$$

The prediction horizon is $\mathcal{N}=\{0,1,2, \ldots, N\}$, with $N$ being the length of the horizon. The optimization variables in (19) are

$$
X=\left\{u_{g, k+j}, u_{g, k+j}^{\mathrm{mpc}}, u_{k+j}^{\mathrm{mpc}}, \hat{x}_{k+j+1 \mid k}^{\mathrm{mpc}}, \hat{z}_{k+j+1 \mid k}^{\mathrm{mpc}}\right\}_{k \in \mathcal{N}} .
$$

As defined by (7), the frequency deviation, $\hat{z}_{\Delta f, k+j \mid k}^{\mathrm{mpc}}$, is available as part of the output vector $\hat{z}_{k+j \mid k}^{\mathrm{mpc}}$, and $\tilde{u}_{g, k+j}^{\mathrm{mpc}}=$ $u_{k+j}^{\mathrm{mpc}}$. The input parameters to (19) are the state space matrices $\left(A, B, E, C_{z}\right)$, the nominal set-point $u_{g, k+j}^{\text {nom }}$, the load forecast correction $d_{k+j}^{\mathrm{mpc}}$, the gain vector $K$, the generation limits $\left(\underline{u}_{k+j}, \bar{u}_{k+j}\right)$, the filtered estimate $\hat{x}_{k \mid k}^{\mathrm{mpc}}$, and the output $\hat{z}_{k \mid k}^{\mathrm{mpc}}$. The stage cost $l_{j}\left(u_{g, k+j}, \hat{z}_{k+j \mid k}\right)$ is defined subsequently.

Equations (19b) and (19c) are the state and output predictions. These constraints are governed by the Kalman filter equations (16). Equations (19d) and (19e) follow from (17) and (18). Equations (19e) limits the generator set-points. The limits are time-varying to account for both generator-specific technical limits, as well as limits that are determined by external factors, e.g. the wind speed for wind turbines.

\section{Objective Function}

The stage cost in the OCP objective function (19a) is defined as

$$
\begin{aligned}
& l_{k}\left(u_{g, k}^{\mathrm{mpc}}, z_{k+1}^{\mathrm{mpc}}\right)=\alpha \phi^{\mathrm{eco}}\left(u_{g, k}^{\mathrm{mpc}}, z_{k+1}^{\mathrm{mpc}}\right) \\
&+(1-\alpha) \phi^{\mathrm{sp}}\left(u_{g, k}^{\mathrm{mpc}}, z_{k+1}^{\mathrm{mpc}}\right), \quad k \in \mathcal{N} .
\end{aligned}
$$

The function $\phi^{\text {eco }}$ is an economic cost function, which is related directly to the cost of operation. The function $\phi^{\mathrm{sp}}$ is a conventional set-point based penalty function. The parameter $\alpha$ is a tuning-parameter to trade-off cost of operation and set-point tracking. In general, the functions $\phi^{\text {eco }}$ and $\phi^{\text {sp }}$ should be designed to fit the particular application. For the application considered in this paper, we use the economic cost function

$$
\begin{aligned}
& \phi^{\mathrm{eco}}\left(u_{g, k}^{\mathrm{mpc}}, z_{k+1}^{\mathrm{mpc}}\right)=r^{T}\left|u_{g, k}^{\mathrm{mpc}}-u_{g, k-1}^{\mathrm{mpc}}\right| \\
& +c^{T} \max \left(z_{g, k+1}^{\mathrm{mpc}}, 0\right)+\bar{c}^{T} \max \left(-z_{g, k+1}^{\mathrm{mpc}}, 0\right) \\
& +\bar{q} \max \left(z_{\Delta f, k+1}^{\mathrm{mpc}}-\bar{f}, 0\right)+\underline{q} \max \left(\underline{f}-z_{\Delta f, k+1}^{\mathrm{mpc}}, 0\right) .
\end{aligned}
$$

The max function and the absolute value function are evaluated element-wise. The frequency deviation, $z_{\Delta f, k+1 \mid k}^{\mathrm{mpc}}$, and the generator outputs, $z_{g, k+1 \mid k}^{\mathrm{mpc}}$, are available as part of the output vector $z_{k+1}^{\mathrm{mpc}}$.

The cost function (21) consists of three terms. The first term, (21a), is an $\ell_{1}$-regularization term on the input-rate. The parameter $r=\left[r_{g_{1}}, r_{g_{2}}, \ldots, r_{g_{M}}\right]$ is a cost vector associated with wear and tear on the generators. The second term, (21b), is related to the cost of generation, $c=$ $\left[c_{g_{1}}, c_{g_{2}}, \ldots, c_{g_{M}}\right]$ for each generator. We define $\bar{c}$, such that $\bar{c}_{i}=1 / c_{g_{i}}$, for $i \in \mathcal{M}$. For upward activation of operational reserves the penalty is $c$, and for downward activation of operational reserves the penalty is $\bar{c}$. We do not use $c$ for downward activation, as the operational reserves should be activated only to compensate for the load not accounted for in the nominal production plan. The final term, (21c), is related to the cost of frequency deviations. The cost $\bar{q}$ is imposed for frequency deviations larger than $\bar{f}$, and the cost $\underline{q}$ is imposed for frequency deviations smaller than $\underline{f}$. The limits $f$ and $\bar{f}$ are the cut-off frequency deviations, at which critical actions such as load shedding are initiated to avoid a blackout. In case the nominal production plan contains frequency deviations, these limits should be modified accordingly.

The set-point based penalty function, $\phi^{\mathrm{sp}}$, is defined as

$$
\begin{aligned}
\phi^{\mathrm{sp}}\left(u_{g, k}^{\mathrm{mpc}}, z_{k+1}^{\mathrm{mpc}}\right)=\left(u_{g, k}^{\mathrm{mpc}}\right)^{T} R^{\mathrm{sp}} & u_{g, k}^{\mathrm{mpc}} \\
& +\left(z_{k+1}^{\mathrm{mpc}}\right)^{T} Q^{\mathrm{sp}} z_{k+1}^{\mathrm{mpc}} .
\end{aligned}
$$

Note that $u_{g, k}^{\mathrm{mpc}}=u_{g, k}-u_{g, k}^{\mathrm{nom}}$, and $z_{k}^{\mathrm{mpc}}=z_{k}-z_{g, k}^{\mathrm{nom}}$, such that (22) penalizes deviations from the nominal production plan.

The problem (19) is formulated as a convex quadratic program. For $\alpha=1$, the quadratic terms (22) drop out of the stage cost (20). In this special case, the optimization problem is a linear program. We solve the OCP using Gurobi [27].

\section{Case Study}

In this section, we test the proposed EMPC scheme using a simulation case study based on the Faroe Islands' power system. The system is a reduced system that consists of $M=$ 4 power generators. The EMPC based controller is compared to set-point based MPC and conventional frequency-based PI-control. The simulations are performed using an Intel(R) Xeon(R) CPU @ 2.67GHz with 12 GB RAM running a 64bit Windows 7 Enterprise operating system.

We consider a time-varying load over 300 seconds. The load is assumed to include a portfolio of non-controllable wind-turbines. In the Faroe Islands, there are several locally 


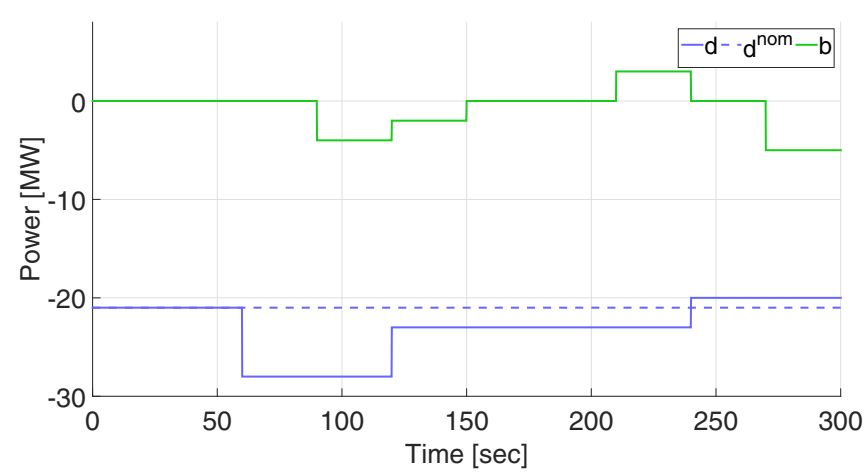

Fig. 4. Case study simulation scenario. The unit-commitment problem is solved based on the nominal load forecast $d^{\text {nom }}$. Updated forecasts and measurements are used in the EMPC scheme to control the system frequency in real-time.

owned wind-turbines that are not controlled by SEV. Fig. 4 shows the scenario set-up.

The nominal load forecast is $d_{k}^{\text {nom }}=-21 \mathrm{MW}$ over the entire simulation scenario. The deviations, $b_{k}$ and $d_{k}^{\mathrm{mpc}}=$ $d_{k}-d_{k}^{\text {nom }}$, from the nominal forecast occur due to fluctuations in the power production and the power consumption. A significant part of the deviations is due to the noncontrollable wind-turbines. The component $d_{k}^{\mathrm{mpc}}$ is predictable during real time control, and the component, $b_{k}$, is estimated based on measurements. In addition to these piece-wise constant deviations, the load and the generators are affected by process noise, as defined in (9). In our simulations the process noise covariance matrix is $R_{w}=I_{4}$, where $I_{4}$ is the identity matrix of size $4 \times 4$. The measurement noise covariance matrix is $R_{v}=\operatorname{blkdiag}\left(0.1 I_{6}, 0\right)$. Thus, only the frequency measurement is noise-free. We have provided the noise covariance matrices for (9), such that the values can be related directly to the physical system. The nominal frequency in the Faroe Islands' power system is $f_{0}=50 \mathrm{~Hz}$.

\section{A. System Parameters}

The case study power generators are modeled as first order systems in the form

$$
Z_{g_{i}}(s)=\frac{1}{\tau_{g_{i}} s+1} U_{g_{i}}(s), \quad i \in \mathcal{M},
$$

where $Z_{g_{i}}$ [MW] is the power production of generator $i$, and $U_{g_{i}}$ [MW] is the power set-point of generator $i$. The load has the similar form

$$
Z_{l}(s)=\frac{1}{\tau_{l} s+1} U_{l}(s),
$$

$\tau_{l}<\tau_{g_{i}}$, for $i \in \mathcal{M}$. We use $\tau_{l}=0.5 \mathrm{~s}$. In (24), $U_{l}(s)$ [MW] is the load set-point, and $Z_{l}(s)$ [MW] is the actual load. The transfer functions (23) and (24) are realized in state space form, to form the system (9).

Table I lists the case study system parameters. The data represents actual generators in the Faroe Islands. Due to confidentiality reasons, the data has been partly modified. Moreover, the inertia provided by each generator is scaled up
TABLE I

CASE STUDY SYSTEM PARAMETERS.

\begin{tabular}{llcccc}
\hline Name & Type & $H_{i}[\mathrm{~s}]$ & $\underline{u}_{i, k}[\mathrm{MW}]$ & $\bar{u}_{i, k}[\mathrm{MW}]$ & $\tau_{i}[\mathrm{~s}]$ \\
\hline Gen. 1 & Hydro & 3.1 & 3 & 20 & 8 \\
Gen. 2 & Hydro & 2.5 & 2 & 6 & 6 \\
Gen. 3 & Diesel & 1.8 & 1 & 5 & 1 \\
Gen. 4 & Diesel & 8.2 & 5 & 15 & 3 \\
\hline
\end{tabular}

to better represent the full scale system. The parameters listed in Table I are constant over the entire simulation scenario. The unit rating, $S_{i}$ [MVA], is defined to have the same magnitude as $\bar{u}_{i, k}$. The primary control gain vector is $K=$ $[20 / 3,2,5 / 3,5]^{T}[\mathrm{MW} / \mathrm{Hz}]$. These gains are computed based on a $6 \%$ speed droop for each of the generators [4]. The power generator production costs in Euro/MWh are 4, 8, 80, and 60 , respectively. Therefore, $c=\bar{T}_{s} / 3600 \cdot[4,8,80,60]^{T}$, where $\bar{T}_{s}=n T_{s}$ is the sampling time of the controller. The prices defined here are similar to the estimates provided in [28]. The input-rate cost is defined to be 0.05 Euro/MW, such that $r=0.05 \cdot[1,1,1,1]^{T}$. The hydro generators have a lower production cost than the diesel generators, but they have limited reservoirs. For this case study, the reservoirs are assumed not to have any limits. Within each generator group, the smaller and faster generator has the highest operating cost.

The nominal set-point is $u_{g, k}^{\text {nom }}=[8,6,1,6]$. These setpoints are computed by solving an economic dispatch problem, considering operational reserve requirements [1], [2]. The simulation is started from steady-state, such that $z_{g, k}^{\text {nom }}=$ $u_{g, k}^{\text {nom }}$. For the disturbance model defined in (13), we use the noise covariance matrix $R_{\eta}=0.1$.

\section{B. Controller}

The controller sampling time is $\bar{T}_{s}=0.5$ seconds, and we define the prediction horizon to be $N=80$ time steps. We have verified in simulations that the closed-loop system is stable for this choice of $N$. The cut-off frequency deviations are $\bar{f}=-f=1 \mathrm{~Hz}$. Frequency deviations larger than $\pm 1 \mathrm{~Hz}$ has a very high cost, as it involves potential load-shedding, cascading generator trips, and ultimately a total blackout. We define the price to be $1000 \mathrm{Euro} /(\mathrm{Hz} \cdot \mathrm{s})$. Accordingly, $\underline{q}=\bar{q}=1000 \bar{T}_{s}$. We note that the economic criterion, (21), may be modified to include several cut-off frequencies with different costs.

The weights in the set-point based criterion (22) are partitioned as $Q^{\mathrm{sp}}=\operatorname{blkdiag}\left(Q_{g}^{\mathrm{sp}}, Q_{l}^{\mathrm{sp}}, Q_{b}^{\mathrm{sp}}, Q_{\Delta f}^{\mathrm{sp}}\right)$, and $R^{\mathrm{sp}}=R_{g}^{\mathrm{sp}}$. We use $Q_{g}^{\mathrm{sp}}=I_{4}, Q_{l}^{\mathrm{sp}}=Q_{b}^{\mathrm{sp}}=0, Q_{\Delta f}^{\mathrm{sp}}=100$, and $R_{g}^{\mathrm{sp}}=I_{4}$. This means that deviations from the nominal frequency have a much higher penalty, compared to generator deviations from their nominal production plan. We scale the weights $Q^{\mathrm{sp}}$ and $R^{\mathrm{sp}}$ by a factor $\bar{T}_{s} / 3600$, such that the economic criterion, (21), and the set-point based criterion, (22), is in a comparable scale.

\section{Simulations}

Closed-loop simulations are performed using the tradeoff specifications $\alpha=0, \alpha=0.5$ and $\alpha=1$. The 


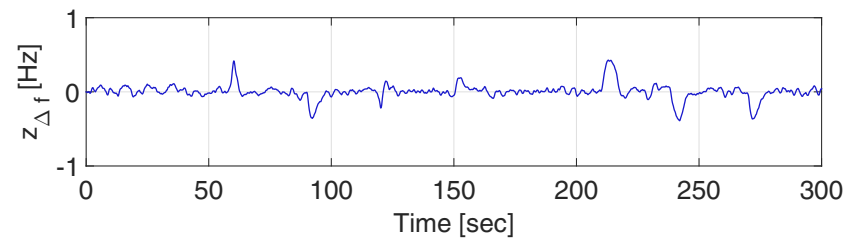

(a) $\operatorname{EMPC}(0)$

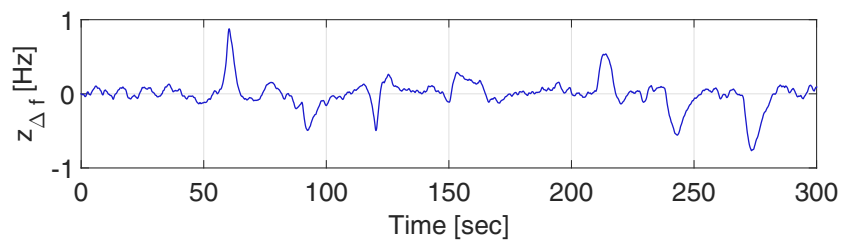

(b) $\operatorname{EMPC}(0.5)$

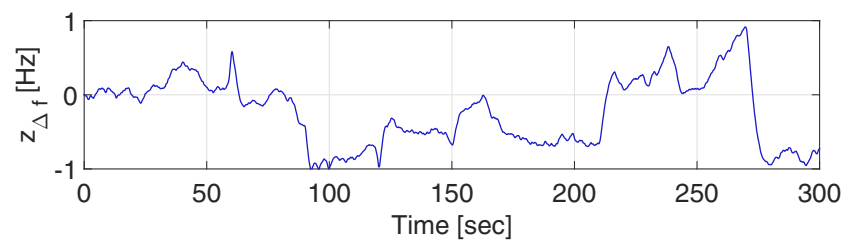

(c) $\operatorname{EMPC}(1)$

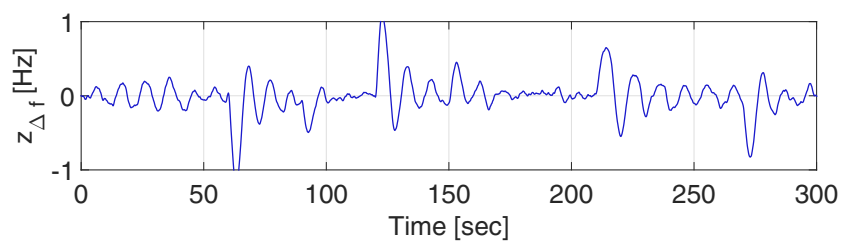

(d) PI-control

Fig. 5. System frequency associated with the simulations in Fig. 6.

case $\alpha=0$ corresponds to set-point based MPC, and $\alpha=1$ only considers cost minimization. The case $\alpha=0.5$ balances the two extreme cases. For compact notation, we use $\operatorname{EMPC}(\alpha)$ to denote EMPC with trade-off parameter $\alpha$. We also include a conventional frequency-based PI-controller in our comparison. We have tuned the PI-controller by trial and error.

Fig. 5 and Fig. 6 illustrate closed-loop simulations for the four different strategies described above. Fig. 5 shows the system frequency, and Fig. 6 shows the power production of the generators

For $\alpha=0$, the EMPC scheme coincides with set-point based MPC. In this case, all the generators with free generation capacity participate in keeping the frequency close to its nominal value. Similar behavior is observed for the PIcontroller. For $\alpha=0.5$, slightly larger frequency deviations are allowed than for set-point based MPC, such that slower and less expensive units can be prioritized over the fast and expensive generators. Note that the frequency deviation never exceeds the cut-off frequency deviations $\pm 1 \mathrm{~Hz}$. For $\alpha=1$, the generators act similar to the case $\alpha=0.5$. The frequency is however, operated close to a cut-off frequency a significant part of the time. Since the controlled system is a stochastic system, EMPC with $\alpha=1$ is high risk strategy. By reducing $\alpha$, the risk is reduced at the expense of the operating cost.
TABLE II

KEY SIMULATION RESULTS: COST OF OPERATION AND FREQUENCY DEVIATIONS FOR DIFFERENT EMPC TRADE-OFF SPECIFICATIONS, AND FOR PI-CONTROL.

\begin{tabular}{lccc}
\hline & Cost of operation & $\min \left\{z_{\Delta f, k}\right\}$ & $\max \left\{z_{\Delta f, k}\right\}$ \\
\hline EMPC $(0)$ & 15.8 & -0.39 & 0.43 \\
EMPC $(0.1)$ & 10.9 & -0.45 & 0.48 \\
EMPC $(0.2)$ & 7.20 & -0.48 & 0.61 \\
EMPC $(0.3)$ & 4.67 & -0.52 & 0.76 \\
EMPC $(0.5)$ & 2.68 & -0.76 & 0.87 \\
EMPC $(1)$ & 2.10 & -1.01 & 0.91 \\
PI-control & 14.2 & -1.19 & 1.10 \\
\hline
\end{tabular}

Table II provides key data from the illustrated simulations, and for additional values of the trade-off parameter $\alpha$. The costs reported in this table are computed as

$$
\pi=\sum_{k} c^{T} z_{g, k+1}^{\mathrm{mpc}}+r^{T}\left|u_{g, k}^{\mathrm{mpc}}-u_{g, k-1}^{\mathrm{mpc}}\right|,
$$

The cost (25) is the actual cost of operation. Compared to the criterion (21b), generator costs can be negative in (25) when operational reserves are activated in the downward direction.

The cost associated with set-point based MPC is approximately 16 Euro. Over the course of one year, the price difference between this strategy and EMPC with $\alpha=0.5$, sums to over 1.3 million Euro, which is approximately $3 \%$ of the revenues generated by SEV in 2012. Although the case $\alpha=1$ results in even lower generation costs, it is disregarded due to its high risk. A systematic method for trading-off cost variance and cost expectation may be achieved for $\alpha=1$, by combining the proposed strategy with mean-variance EMPC [29], [30].

\section{CONCLUSIONS}

We develop a novel economic model predictive control scheme for frequency control in a single-area power system. The scheme is a generalization of set-point based MPC, that trades off cost minimization and set-point tracking. Simulations based on a Faroe Islands case study show that the proposed controller reduces cost of operation by almost an order of magnitude, while maintaining a high quality frequency control.

\section{ACKNOWLEDGEMENT}

This work was funded in part by 1) the Danish Ministry of Higher Education and Science in the industrial $\mathrm{PhD}$ project "Stochastic MPC with Applications in Smart Energy Systems" (11-117435); and 2) The Danish Council for Strategic Research in the project "CITIES - Centre for IT-Intelligent Energy Systems in Cities" (1305-00027B).

\section{REFERENCES}

[1] J. F. Restrepo and F. D. Galiana, "Unit Commitment With Primary Frequency Regulation Constraints," IEEE Transactions on Power Systems, vol. 20, no. 4, pp. 1836-1842, 2005.

[2] M. Carrión and J. M. Arroyo, "A computationally efficient mixedinteger linear formulation for the thermal unit commitment problem," IEEE Transactions on Power Systems, vol. 21, no. 3, pp. 1371-1378, 2006. 


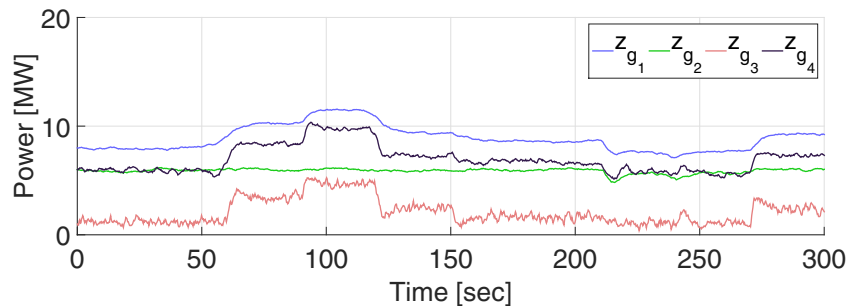

(a) $\operatorname{EMPC}(0)$

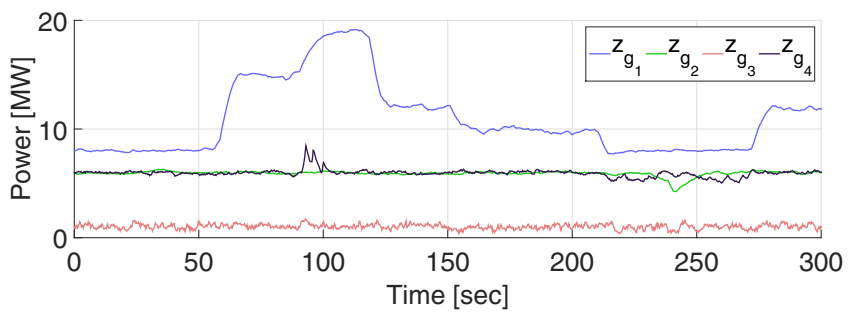

(c) $\operatorname{EMPC}(1)$

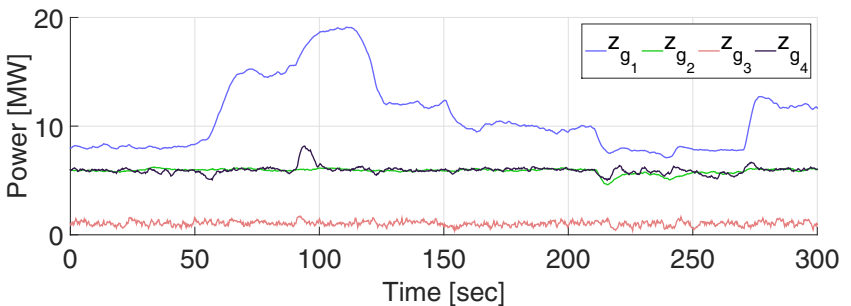

(b) $\operatorname{EMPC}(0.5)$

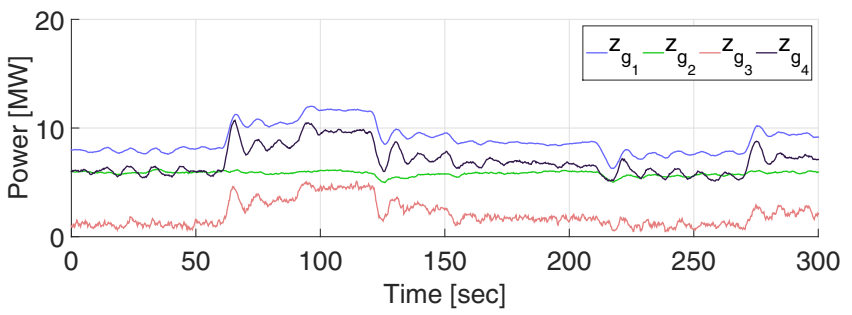

(d) PI-control

Fig. 6. Closed-loop simulation for EMPC and frequency-based PI-control. The EMPC scheme is tested using the trade-off specifications $\alpha=0, \alpha=0.5$, and $\alpha=1$.

[3] J. Kumar, K.-H. Ng, and G. Sheble, "AGC simulator for price-based operation. I. A model," IEEE Transactions on Power Systems, vol. 12, no. 2, pp. 527-532, 1997.

[4] H. Bevrani, Robust Power System Frequency Control. New York: Springer-Verlag, 2009.

[5] G. Andersson, "Dynamics and Control of Electric Power Systems," Lecture Notes in Electrical Engineering, Power Systems Laboratory, ETH Zurich, Tech. Rep., 2012. [Online]. Available: http://eeh.ee.ethz.ch

[6] I. Ibraheem, P. Kumar, and D. P. Kothari, "Recent philosophies of automatic generation control strategies in power systems," IEEE Transactions on Power Systems, vol. 20, no. 1, pp. 346-357, 2005.

[7] D. Angeli, "Economic Model Predictive Control," in Encyclopedia of Systems and Control. Springer London, 2014, pp. 1-9.

[8] M. Ellis, H. Durand, and P. D. Christofides, "A tutorial review of economic model predictive control methods," Journal of Process Control, vol. 24, no. 8, pp. 1156-1178, 2014.

[9] D. J. Trudnowski, W. L. McReynolds, and J. Johnson, "Real-time very short-term load prediction for power-system automatic generation control," IEEE Transactions on Control Systems Technology Technology, vol. 9, no. 2, pp. 254-260, 2001.

[10] N. Atic, D. H. A. Rerkpreedapong, and A. Feliachi, "NERC compliant decentralized load frequency control design using model predictive control," in 2003, IEEE Power Engineering Society General Meeting, 2003, pp. 554-559.

[11] L. Kong and L. Xiao, "A New Model Predictive Control SchemeBased Load-Frequency Control," in IEEE International Conference on Control and Automation, 2007, 2007, pp. 2514-2518.

[12] D. Rerkpreedapong, N. Atic, and A. Feliachi, "Economy oriented model predictive load frequency control," in 2003 Large Engineering Systems Conference on Power Engineering, 2003, pp. 12-16.

[13] F. Abbaspourtorbati, M. Scherer, A. Ulbig, and G. Andersson, "Towards an optimal activation pattern of tertiary control reserves in the power system of Switzerland," in American Control Conference (ACC), 2012, 2012, pp. 3629-3636.

[14] A. N. Venkat, I. A. Hiskens, J. B. Rawlings, and S. J. Wright, "Distributed MPC Strategies With Application to Power System Automatic Generation Control," IEEE Transactions on Control Systems Technology, vol. 16, no. 6, pp. 1192-1206, 2008.

[15] T. H. Mohamed, H. Bevrani, A. A. Hassan, and T. Hiyama, "Decentralized model predictive based load frequency control in an interconnected power system," Energy Conversion and Management, vol. 52, no. 2, pp. 1208-1214, 2011.

[16] M. Ma, H. Chen, X. Liu, and F. Allgöwer, "Distributed model predictive load frequency control of multi-area interconnected power system," International Journal of Electrical Power \& Energy Systems, vol. 62, no. 0, pp. 289-298, 2014.
[17] Twenties, "Providing Flexibility with a Virtual Power Plant," Tech Rep., 2013. [Online]. Available: http://twenties-project.eu/node/18

[18] M. Maasoumy, A. Sanandaji, B. M. Sangiovanni-Vincentelli, and K. Poolla, "Model Predictive Control of regulation services from commercial buildings to the smart grid," in 2014 American Control Conference (ACC), 2014, pp. 2226-2233.

[19] J. Carpentier, "'To be or not to be modern' that is the question for automatic generation control (point of view of a utility engineer)," International Journal of Electrical Power \& Energy Systems, vol. 7 , no. 2, pp. 81-91, 1985 .

[20] A. S. Debs, Modern Power Systems Control and Operation. Springer US, 1988

[21] A. J. Wood and B. F. Wollenberg, Power Generation, Operation, and Control, 3rd ed. John Wiley \& Sons, 2013.

[22] K. Edlund, T. Mølbak, and J. D. Bendtsen, "Simple models for model-based portfolio load balancing controller synthesis," in 6th IFAC Symposium on Power Plants and Power Systems Control, 2009, pp. 173-178.

[23] P. Kundur, N. J. Balu, and M. G. Lauby, Power System Stability and Control, ser. EPRI power system engineering series. McGraw-Hill, 1994.

[24] R. Kalman, "A New Approach to Linear Filtering and Prediction Problems," Transactions of the ASME, Journal of Basic Engineering, vol. 82, no. Series D, pp. 35-45, 1960.

[25] G. Pannocchia and J. B. Rawlings, "Disturbance Models for OffsetFree Model-Predictive Control," AIChE Journal, vol. 49, no. 2, pp. 426-437, 2003.

[26] F. Borrelli and M. Morari, "Offset Free Model Predictive Control," in 2007 46th IEEE Conference on Decision and Control (CDC), 2007, pp. $1245-1250$.

[27] Gurobi Optimization Inc., "Gurobi Optimizer Reference Manual," 2015. [Online]. Available: http://www.gurobi.com

[28] EIA, "Levelized Cost and Levelized Avoided Cost of New Generation Resources in the Annual Energy Outlook 2014," U.S. Energy Information Administration, Tech. Rep., 2014. [Online]. Available: http://eia.gov/forecasts/aeo/er/index.cfm

[29] L. E. Sokoler, B. Dammann, H. Madsen, and J. B. Jørgensen, "A Mean-Variance Criterion for Economic Model Predictive Control of Stochastic Linear Systems," in 2014 IEEE 53rd Annual Conference on Decision and Control (CDC), 2014, pp. 5907-5914.

[30] —, "A Decomposition Algorithm for Mean-Variance Economic Model Predictive Control of Stochastic Linear Systems," in 2014 IEEE Multi-conference on Systems and Control, 2014, pp. 1086-1093. 